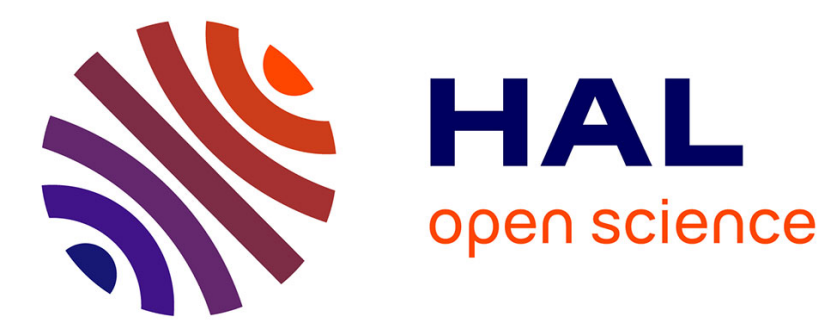

\title{
Adjustment of muscle coordination during an all-out sprint cycling task
}

Gaël Guilhem, Sylvain Dorel, Antoine Couturier, François Hug

\section{To cite this version:}

Gaël Guilhem, Sylvain Dorel, Antoine Couturier, François Hug. Adjustment of muscle coordination during an all-out sprint cycling task. Medicine and Science in Sports and Exercise, 2012, 44 (11), pp.2154-2164. 10.1249/MSS.0b013e3182625423 . hal-01616637

\section{HAL Id: hal-01616637 \\ https://hal-insep.archives-ouvertes.fr/hal-01616637}

Submitted on 9 Oct 2018

HAL is a multi-disciplinary open access archive for the deposit and dissemination of scientific research documents, whether they are published or not. The documents may come from teaching and research institutions in France or abroad, or from public or private research centers.
L'archive ouverte pluridisciplinaire HAL, est destinée au dépôt et à la diffusion de documents scientifiques de niveau recherche, publiés ou non, émanant des établissements d'enseignement et de recherche français ou étrangers, des laboratoires publics ou privés. 


\title{
Adjustment of Muscle Coordination during an All-Out Sprint Cycling Task
}

\author{
SYLVAIN DOREL ${ }^{1,2}$, GAEL GUILHEM $^{2}$, ANTOINE COUTURIER $^{2}$, and FRANÇOIS HUG ${ }^{1}$

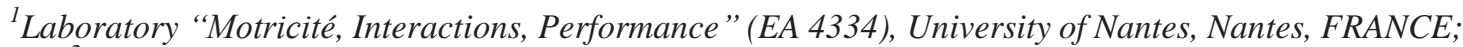 \\ and ${ }^{2}$ Research Department, National Institute for Sports (INSEP), Paris, FRANCE
}

\section{ABSTRACT}

\begin{abstract}
Purpose: This study was designed to assess muscle coordination during a specific all-out sprint cycling task (Sprint). The aim was to estimate the EMG activity level of each muscle group by referring to the submaximal cycling condition (Sub150 W) and to test the hypothesis that a maximal activity is reached for all of the muscles during Sprint. Methods: Fifteen well-trained cyclists were tested during submaximal and sprint cycling exercises and a series of maximal voluntary contractions (MVCs) in isometric and isokinetic modes (MVC at the three lower limb joints). Crank torque and surface EMG signals for 11 lower limb muscles were continuously measured. Results: Results showed that Sprint induced a very large increase of EMG activity level for the hip flexors (multiplied by 7-9 from $150 \mathrm{~W}$ to Sprint) and the knee flexors and hip extensors (multiplied by $5-7$ ), whereas plantar flexors and knee extensors demonstrated a lower increase (multiplied by 2-3). During Sprint, EMG activity level failed to reach a maximal value for hamstrings, tibialis anterior, tensor fasciae latae, and gluteus maximus (i.e., < $70 \%$ to $80 \%$ of peak EMG activity during MVC, $P<0.05$ to $P<0.001$ ), and individual EMG patterns demonstrated a significant earlier onset and/or later offset for the majority of the muscles $(P<0.01$ to $P<0.001)$. Conclusions: Results clearly suggest a change in the relative contribution of the different muscles to the power production between Sub150 W and Sprint, and provide evidence that EMG activ- ity level is not systematically maximal for all muscles involved in the all-out sprint cycling task. The longer period of activity induced during Sprint is likely to represent an interesting coordination strategy to enhance the work generated by all of the muscle groups. Key Words: PEDALING, MAXIMAL POWER OUTPUT, ELECTROMYOGRAPHY, NORMALIZATION, MULTIJOINT TASK, MAXIMAL VOLUNTARY CONTRACTION
\end{abstract}

$\mathrm{M}$ uscle coordination is defined as "a distribution of activation or force among individual muscles to produce given combination of joint moment", (22). This has been widely studied during pedaling, especially the alteration of EMG activity of lower limb muscles in response to changes in pedaling rate, power output, and body posture (for review, see Ref. [15]). Among these parameters, it is well established that increased power output is one of the most important adjustment factors responsible for a global increase of joint load and hence muscle activity level $(9,15)$. Some studies have reported that changes in muscle activity level in response to increase of power output conditions differ between different lower limb muscles $(14,18,25,29)$. For instance, although gastrocnemius activity level is relatively stable up to $50 \%-60 \%$ of maximal aerobic

Address for correspondence: Sylvain Dorel, Ph.D., Laboratory "Motricité, Interactions, Performance" (EA 4334), University of Nantes, 25 bis boulevard Guy Mollet, BP 72206, 44322 Nantes cedex 3, France; E-mail: sylvain.dorel@ univ-nantes.fr.

DOI: 10.1249/MSS.0b013e3182625423 power and then moderately increases $(14,18,20)$, the activity level of rectus femoris (RF), semimembranosus (SM), and gluteus maximus (GMax) is much more influenced by the workload level (9). However, these studies have focused on submaximal pedaling exercises (i.e., intensity below the maximal aerobic power).

Literature concerning muscle coordination during supramaximal cycling exercise is scarce, especially regarding the all-out sprint exercise. Hug et al. (16) recently explored the effect of different mechanical constraints on muscle synergies during pedaling. Their results showed that extreme changes in torque at a given pedaling rate (i.e., submaximal vs. sprint exercise) did not modify the composition of the extracted muscle synergies despite an observed slight adaptation in their activation timing. In this work, muscle synergies were defined as low-dimensional modules formed by muscles activated in synchrony (30), where the output level of this activation was not taken into consideration. Because some authors (32) have suggested that timing and amplitude of EMG patterns may be controlled independently, the nature of changes in muscle activity level induced by all-out sprint pedaling exercise remains an open question. The few studies that have investigated muscle activity level during such a task focused on a small number of muscles (two and five muscles in Samozino et al. [26] and Hautier et al. [11], respectively). Moreover, authors did not attempt to estimate the degree of activation of each muscle, which requires to 
refer to the maximal activity level obtained during a maximal voluntary contraction (i.e., MVC normalization procedure $[3,9,24])$. To the best of our knowledge, only Fernandez-Pena et al. (10) examined the change in EMG activity level from submaximal to all-out sprint isokinetic cycling, but, once again, only four muscles were considered. In addition, EMG activity levels were expressed as referring to the all-out sprint cycling condition. As discussed by Hug and Dorel (15), this kind of normalization procedure does not ensure that the maximal level of activation was reached for all of the muscles, precluding precise interpretations in terms of degree of muscle activity.

Considering all of these elements, it remains unclear to what extent the activity level of individual lower limb muscles increases, and whether it actually reaches an absolute maximal level during an all-out sprint cycling task. Interestingly, on the basis of mechanical data, recent studies showed important adaptations to supramaximal compared with submaximal pedaling exercises, such as a dominant power-producing action of the hip extension and a higher relative knee flexion power $(8,21)$. This clearly suggests modification of activity (level and/or timing) of the muscles involved in these actions.

Therefore, the present study aims to investigate muscle coordination during the all-out sprint cycling task. The first purpose was to quantify changes in both level and timing aspects of EMG activity of the lower limb muscles during this supramaximal exercise compared with the traditional submaximal cycling condition. On the basis of the aforementioned studies $(8,21)$, we hypothesized that the sprint condition would mainly induce a larger increase of activity level for muscles involved in knee flexion and hip extension. The second aim was to determine whether maximal muscle activity is reached during the all-out sprint cycling task. We tested the assumption classically accepted in the literature $(10,24)$ that all-out sprint cycling would allow all of the mono- and biarticular muscles to be maximally recruited. For these purposes, we measured the EMG activity of 11 surface lower limb muscles during an all-out sprint isokinetic cycling exercise and two exercises performed at a submaximal level (i.e., $150 \mathrm{~W}$ and $80 \%$ of maximal aerobic power). A series of monoarticular MVCs was performed as a normalization procedure for each muscle to quantify the degree of muscle activity.

\section{METHODS}

\section{Participants}

Fifteen elite sprint cyclists volunteered to participate in this study: 5 women (age, $21.8 \pm 2.9 \mathrm{yr}$; body mass, $62.6 \pm$ $4.2 \mathrm{~kg}$; height, $167.4 \pm 6.1 \mathrm{~cm}$ ) and $10 \mathrm{men}$ (age: $22 \mathrm{~T} 2.9 \mathrm{yr}$; body mass: $84.0 \pm 5.7 \mathrm{~kg}$; height: $177.4 \pm 7.4 \mathrm{~cm})$. The test procedures were explained to the participants before they gave their written informed consent. The experimental design of the study was approved by the local Ethical
Committee of Saint-Germain-en-Laye (France) (acceptance number 06016) and was carried out in accordance with the Declaration of Helsinki. All subjects were highly trained and competed in international track cycling events. The study was performed during the racing season.

\section{Exercise Protocol}

Each participant performed two tests separated by 3-6 d and carried out at the same time of the day, at least $3 \mathrm{~h}$ after a meal. During a first session, they were asked to perform a torque-velocity test on a cycle ergometer (three cycling sprints of $5 \mathrm{~s}$ interspaced by $8 \mathrm{~min}$ of recovery) according to the protocol proposed by Dorel et al. (6). This session aimed at determining specific power-velocity characteristics: the maximum power $\left(P_{\max }\right)$ and the corresponding specific optimal pedaling rate $\left(f_{\text {opt }}\right)$ at which $P_{\max }$ occurred (for details, see Ref. [6]). The second session was separated into two parts: a maximal voluntary isometric and isokinetic contractions protocol and a series of cycling exercises.

MVC procedure. Subjects began with tests that consisted of several MVCs in isometric $\left(\mathrm{MVC}_{\mathrm{Isom}}\right)$ and isokinetic $\left(\mathrm{MVC}_{\mathrm{Isokin}}\right)$ situations. This procedure was carried out on a Con-Trex isokinetic ergometer (CMV AG, Dübendorf, Switzerland). Isometric MVCs (3-s duration) were performed in the sagittal plane: flexion (FLEX) and extension (EXT) for the three joints of the right lower limb by fixing a constant angle: hip ( $45^{\circ}$ in EXT, $60^{\circ}$ in FLEX), knee $\left(70^{\circ}\right.$ in EXT, $50^{\circ}$ in FLEX) and ankle ( $0^{\circ}$ in EXT, $-10^{\circ}$ in FLEX). These angles were determined in accordance with the range of the angle values classically reported at which maximal torque and EMG activity are produced $(9,24)$. During isokinetic contractions, the velocity was set at $30^{\circ} \cdot \mathrm{s}^{-1}$, and the same range of motion was fixed for EXT and FLEX: $20^{\circ}$ to $80^{\circ}$ for the hip, $30^{\circ}$ to $80^{\circ}$ for the knee $\left(0^{\circ}=\right.$ full extended position $)$, and $-20^{\circ}$ to $5^{\circ}$ for the ankle $\left(0^{\circ}=\right.$ mid ankle position and neg- ative angle in direction of plantarflexion). Figure 1 depicts the global standardized body positions used for the six muscle groups tested. For each joint, the session started with a warm-up of 20 (for hip and knee) or 10 (for ankle) progressive concentric contractions in flexion and extension from submaximal up to quasimaximal involvement, and then the MVC efforts were performed as follows: four $\mathrm{MVC}_{\text {Isom }}$ (two FLEX and two EXT alternatively with 60-s recovery in between) and a series of three $\mathrm{MVC}_{\text {Isokin }}$ (alternating FLEX and EXT without recovery). A 5-min recovery period was imposed between $\mathrm{MVC}_{\text {Isom }}$ and $\mathrm{MVC}_{\text {Isokin }}$ and 10-min pe- riod between the MVC protocols of the three different joints (i.e., hip, knee, and ankle). Mechanical signals (i.e., torque, velocity, and position) were recorded at a sampling fre- quency of $250 \mathrm{~Hz}$.

Submaximal and all-out sprint cycling exercises. The second part of this session consisted of a submaximal cycling exercise and a series of brief all-out cycling exercises performed in an isokinetic mode. After a warm-up (6 min at $100 \mathrm{~W})$, subjects were asked to perform two 

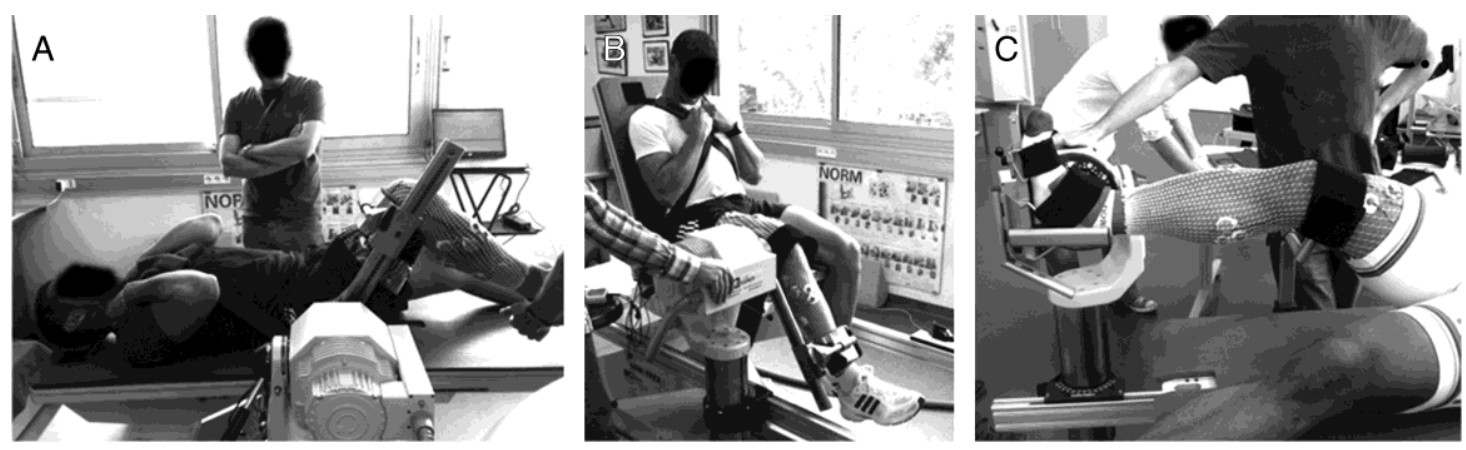

FIGURE 1-The testing conditions and global body posture retained for the MVC efforts on the Con-Trex ergometer. Here shown are the specific examples of isometric MVC for the hipflexion (in $60^{\circ}$ hipflexion position (A), thekneeflexion (in $50^{\circ}$ kneeflexion position) (B), and the dorsiflexion (supine with $0^{\circ} \mathrm{mid}$ ankle position) (C). For the knee and hip angular positions, $0^{\circ}$ is when the joints are fully extended.

submaximal 3-min exercises at $150 \mathrm{~W}(\operatorname{Sub} 150 \mathrm{~W})$ and $310 \mathrm{~W}$ for the men and $220 \mathrm{~W}$ for the women (SubVT2), both at a constant pedaling rate corresponding to $80 \%$ of $\mathrm{N}_{\text {opt }}$. These values of power and pedaling rate $(100 \pm 4 \mathrm{rpm}$ in this group) were chosen for comparison with the traditional submaximal conditions reported in the literature: a low value of power (i.e., Sub150 W) and a power corresponding to the estimated second ventilatory threshold or $80 \%$ of the maximal aerobic power of the subjects (i.e., SubVT2) $(7,27)$. Then, five all-out 6-s isokinetic sprint exercises at $60 \%, 80 \%, 100 \%$, $120 \%$, and $140 \%$ of $f_{\text {opt }}$ were performed in the seated position, and a last exercise was completed in the standing position (at $80 \% f_{\text {opt }}$ ) as previously described (16). The cycling bouts were interspaced by a complete 8 -min recovery period, and the sprints order was randomized. For the purpose of this study, only the results concerning the seated sprint at $80 \%$ $f_{\text {opt }}$ (Sprint) was examined and compared with both submaximal exercises performed at the same pedaling rate. Before the maximal sprint effort, the flywheel was set at the target velocity to reduce the acceleration phase and to make it possible to briefly reach the isokinetic condition of maximal pedaling without fatigue.

\section{Mechanical and EMG Data Acquisition}

Participants exercised on an electronically braked cycle ergometer (Excalibur Sport; Lode ${ }^{\circledR}$, The Netherlands) equipped with standard cranks (length $=170 \mathrm{~mm}$ ). For each participant, positions of the saddle and handlebar were adjusted to ensure the usual racing position of the participants. Their feet were fixed on their own clipless pedals. The torque exerted on the left and right cranks was measured by strain gauges in the crank arms of the cycle ergometer. The crank angle and the angular velocity were calculated (time derivative) on the basis of transistor-transistor logic rectangular pulses delivered each $2^{\circ}$ by the ergometer. An additional transistor-transistor logic pulse was used to detect the top dead center (TDC) (i.e., highest position of the right pedal with crank arm angle at $0^{\circ}$ ).

The surface EMG of 11 muscles of the right lower limb was recorded continuously throughout the MVC and cycling experimental sessions: GMax, SM, long head of biceps femoris (BF), tensor fasciae latae (TF), vastus medialis (VM), $\mathrm{RF}$, vastus lateralis (VL), gastrocnemius medialis (GM) and lateralis (GL), soleus (SOL), and tibialis anterior (TA). The EMG recording procedure was detailed elsewhere in a previous study (16). Briefly, for each muscle, a pair of surface electrodes was attached to the skin and located according to the recommendations of SENIAM when available (surface EMG for noninvasive assessment of muscles) (12). Raw EMG signals were amplified, simultaneously digitized with TDC pulses (during cycling exercise) at a sampling rate of $1 \mathrm{kHz}$ (ME6000P16; MEGA Electronics Ltd. ${ }^{\circledR}$, Finland), high-pass filtered (20 Hz, Butterworth filter), and root mean squared (RMS) with a 25-ms moving rectangular window.

\section{Data Processing}

All data were analyzed with ${ }_{\mathscr{\Theta}}$ ustom written scripts (Origin 8.5; OriginLab Corporation ， Northampton, MA). EMG and torque signals were smoothed with an antialiasing filter where the cutoff frequency was dynamically computed according to the Shannon theorem (zero lag low-pass filtered: $24 \mathrm{~Hz}$ ). During both $\mathrm{MVC}_{\text {Isom }}$ and $\mathrm{MVC}_{\text {Isokin, the }}$ time window of analysis corresponding to the plateau of the burst (excluding the beginning and the end of contraction) was used to determine the peak EMG RMS value for each muscle (mean maximal value over a period of $200 \mathrm{~ms}$ ). The highest peak EMG amplitude among the two conditions was selected as the reference value representing the maximal neural drive obtained during MVC tests $\left(\mathrm{RMS}_{\text {isomax }}\right)$. The procedure including the maximal isokinetic condition $\left(\mathrm{MVC}_{\text {isokin }}\right)$ was justified to take into account the suggested effect of the joint angle and muscle length on the maximal EMG response $(3,24)$. For information, the distribution of the $\mathrm{RMS}_{\text {isomax }}$ obtained for each muscle either in isometric or isokinetic condition (i.e., number of subjects demonstrating $\mathrm{RMS}_{\text {isomax }}$ during $\mathrm{MVC}_{\mathrm{Isom}} /$ number of subjects demonstrating $\mathrm{RMS}_{\text {isomax }}$ during $\mathrm{MVC}_{\text {Isokin }}$ ) was as follows: TA 2/13, SOL 11/4, GM 4/11, GL 11/4, VL 12/3, VM 13/2, RF 4/11, TF 3/12, BF 2/13, ST 1/14, and GMax 10/5.

During pedaling exercises, a linear interpolation technique was used to obtain a value of torque and EMG RMS 
each degree of rotation. Then, the torque and EMG RMS linear envelope for each muscle were, respectively, averaged

A time (s)
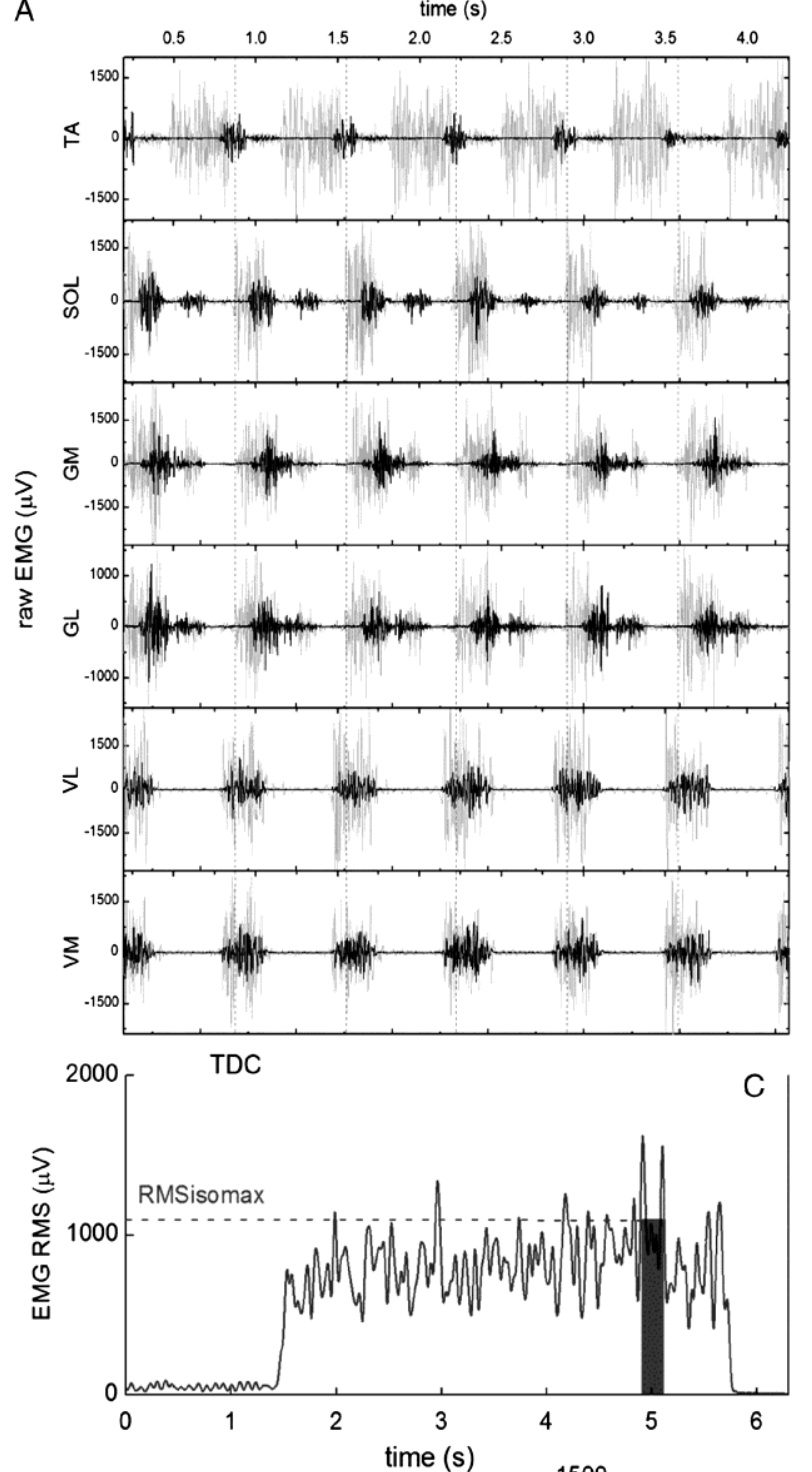

over 30 consecutive pedaling cycles for both Sub150 W and SubVT2 and 7 consecutive cycles for Sprint to get a
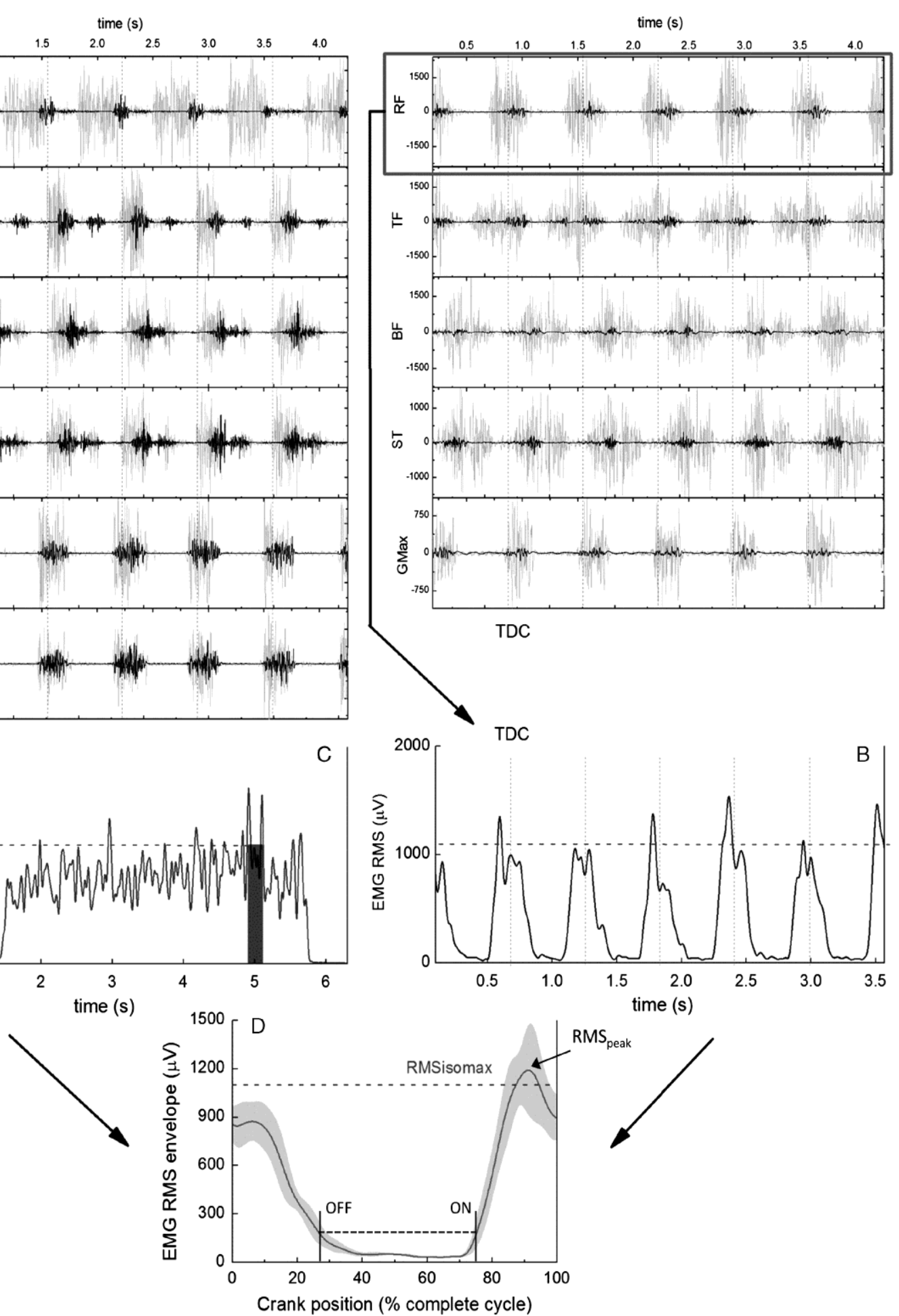

FIGURE 2-Example of EMG signal processing to determine the lower limb muscle activity levels and the study of their activation patterns during the different pedaling conditions. A, Typical example of raw EMG data obtained in Sub 150 W (inblack) and during Sprint (ingray) for the 11 muscles:TA (tibialisanterior), SOL(soleus), and TF (tensorfascialatae). B, EMG data are RMS with a time averaging period of $25 \mathrm{~ms}$ and smoothed to produce alinear envelope of RF (rectus femoris) in Sprint condition. C, Maximal level of activity for RF is determined on the basis of EMG RMS recording during a leg extension MVC $\left(\mathrm{RMS}_{\text {isomax }}=\right.$ mean maximal value over a period of $200 \mathrm{~ms}$ : filled zone). D, Data are averaged over six consecutive pedaling cycles to get a

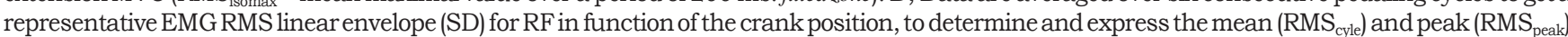
activity levels as a percentage of $\mathrm{RMS}_{\text {isomax }}$, and to obtain the period of muscle activity (ON, onset; OFF, offset; horizontal blackdashed line, threshold at $\left.20 \%\right)$. $\mathrm{TDC}=$ highest pedal position $=0$ - . The gray dashed horizontal line on C and D refers to $\mathrm{RMS}_{\text {isomax }}$ determined in C. GL, gastroncnemius lateralis; GM, gastrocnemius medialis; VL, vastus lateralis; VM, vastus medialis; BF, biceps femoris; SM, semimembransus; GMax, gluteus maximus. 
representative torque and EMG profile for each subject and each condition. These values were plotted against the crank arm angle (0-, TDC, and 180-, bottom dead center (BDC)). Then, the activity level was identified by both determining the maximal EMG RMS value during the burst (i.e., peak value of the EMG pattern, $\mathrm{RMS}_{\text {peak }}$ ) and the mean value over the complete cycle $\left(\mathrm{RMS}_{\text {cycle }}\right)$. The EMG timing analysis consisted of determining the onset $(\mathrm{ON})$ and offset (OFF) of the burst of activity, which was defined as the period where the signal was above a threshold of $20 \%$ of the difference between peak and baseline $(2,5,15)$. In the few cases where two bursts by cycle were detected for some muscles (i.e., TA, GL, GM, SOL, RF, and TF), each pattern was visually checked. When the period between the two bursts was lower than 15- of crank rotation, they were considered as one global burst with the onset corresponding to $\mathrm{ON}$ of the first burst and the offset to OFF of the second burst. In the other cases (i.e., when two really distinct bursts were observed, especially for TA), only the burst common to all subjects was considered. Figure 2 depicts a typical example of recorded raw EMG data for all muscles in the Sub150 W and Sprint conditions, and illustrates the entire EMG data process for one muscle (i.e., RF).

\section{Statistical Analysis}

All analyses were performed using the statistical package STATISTICA (StatSoft, Tulsa, OK) and ORIGIN 8.5 (Origin 8.5, OriginLab Corporation). Data distributions were first checked by the Shapiro-Wilk normality test. All data being normally distributed, the homogeneity of variance between each sample of data was checked using the Levene test. A two-way ANOVA with repeated measures was used to test the effect of power conditions ("power effect," first factor in repeated measures: Sub150 W, SubVT2, and Sprint), the difference between the muscles ("muscle effect," second factor), and their possible interaction ("power $x$ muscle") on muscle activity levels $\left(\mathrm{RMS}_{\text {peak }}\right.$ and $\mathrm{RMS}_{\text {cycle }}$, expressed as the $\left.\% \mathrm{RMS}_{\text {isomax }}\right)$. One-way ANOVA with repeated measures was used to test the effect of the three power conditions on EMG timing variables (onset, offset, and burst duration) for each muscle. When significant $F$-ratios were found, all of the means were compared using a post hoc analysis (Tukey method). Because the raw EMG data are not directly comparable between different muscles, we tested specifically whether maximal muscle activity was reached during the allout sprint cycling for each muscle using a Student's paired $t$-test comparing the mean value of $\mathrm{RMS}_{\text {peak }}$ and $\mathrm{RMS}_{\text {isomax }}$. Values are reported as mean \pm SD throughout the text and the figures. A $P$ value below 0.05 was considered significant.

\section{RESULTS}

The right crank torque profile obtained in the submaximal and all-out sprint conditions is depicted in Figure 3. Mean torque achieved during Sub150 W, SubVT2, and Sprint was
$7.1 \pm 0.8,14.4 \pm 2.4$, and $64.7 \pm 14.1 \mathrm{NIm}$, respectively. The total maximal power output (i.e., with both legs) achieved during the all-out sprint exercise was $1372 \pm 326 \mathrm{~W}(1571 \pm$ $188 \mathrm{~W}$ for men and $974 \pm 97 \mathrm{~W}$ for women).

The two-way repeated-measures ANOVA revealed the main effects of power $(P<0.001)$, and muscle $(P$ G 0.001$)$, on both $\mathrm{RMS}_{\text {peak }}$ and $\mathrm{RMS}_{\text {cycle. A significant interaction }}$ muscle $\mathrm{X}$ power was also found $(P<0.001)$, which permitted understanding of the detailed (by post hoc Tukey analysis) changes in EMG activity level with power conditions for each individual muscle and to show for each of these conditions the differences in EMG activity level among the 11 muscles. For each muscle, the averaged EMG patterns obtained for both submaximal and all-out sprint conditions are depicted in Figure 3, and complete results are detailed below.

Submaximal conditions. Increased power from Sub150 W to SubVT2 induced a significant increase in $\mathrm{RMS}_{\text {peak }}$ for TA, SOL, GM, GL, VL, VM, and GMax ( $P<0.05$, Fig. 4). No significant change was observed for the other muscles. For both submaximal conditions, heterogeneity appeared among the different muscles regarding $\mathrm{RMS}_{\text {peak }}$ expressed as a percentage of $\mathrm{RMS}_{\text {isomax }}$. More precisely, muscles crossing the knee and the ankle (i.e., SOL, GM, GL, VM, and VL) were significantly more activated than muscles crossing the hip (RF, TF, BF, SM, and GMax $)(P<0.001$; Fig. 4). In Sub150 W (i.e., the "reference" submaximal condition compared with the all-out sprint condition), $\mathrm{RMS}_{\text {peak }}$ values ranged from $29 \% \pm$ $8 \%$ (SOL) to $46 \% \pm 16 \%$ (GL) for the muscle group crossing the knee and the ankle and from $11 \% \pm 5 \%$ (RF and TF) to $15 \% \pm 5 \%(\mathrm{SM})$ for the group crossing the hip. None of the variables representing muscle activation timing (i.e., onset, offset, and duration of the burst) was significantly altered between Sub150 W and SubVT2 (Figs. 3 and 5).

All-out sprint versus submaximal conditions. All of the investigated muscles showed a significantly higher $\mathrm{RMS}_{\text {peak }}$ and $\mathrm{RMS}_{\text {cycle }}$ value during the all-out sprint condition compared with both Sub150 W and SubVT2 $(P<$ 0.001 , Figs. 3 and 4 ). Figure 6 depicts the magnitude of these changes in the all-out sprint condition compared with Sub150 W for all of the muscles. A large discrepancy was observed in this increase among the muscles, that is, from X2.1 (for GL) to $\times 9.0$ (for RF) higher $\mathrm{RMS}_{\text {peak }}$ value and from $\times 3.3$ (for GL) to $\times 9.5$ (for $\mathrm{RF}$ ) higher $\mathrm{RMS}_{\text {cycle }}$ value in all-out sprint compared with Sub150 W.

During the all-out sprint condition, one muscle demonstrated a higher $\mathrm{RMS}_{\text {peak }}$ than $\mathrm{RMS}_{\text {isomax }}(\mathrm{SOL}: 126.7 \% \pm$ $33.0 \%$ ), five muscles exhibited an $\mathrm{RMS}_{\text {peak }}$ nonsignificantly different from the $\mathrm{RMS}_{\text {isomax }}(\mathrm{GM}, \mathrm{GL}, \mathrm{VL}, \mathrm{VM}$, and RF; ranged from $91.9 \% \pm 24.6 \%$ for $\mathrm{VM}$ to $104.2 \% \pm 31.1 \%$ for VL, Fig. 4), whereas the others had a significantly lower $\mathrm{RMS}_{\text {peak }}$ than $\mathrm{RMS}_{\text {isomax }}$, that is, a nonmaximal level of activity (TA, TF, BF, SM, and GMax; ranged from $60.3 \% \pm$ $17.7 \%$ for SM, $P<0.001$, to $80.9 \% \pm 24.6 \%$ for $\mathrm{TF}$, $P<0.05$, Fig. 4).

As observed on the EMG patterns (Fig. 3), most of the muscles exhibited significant modifications of their timing 


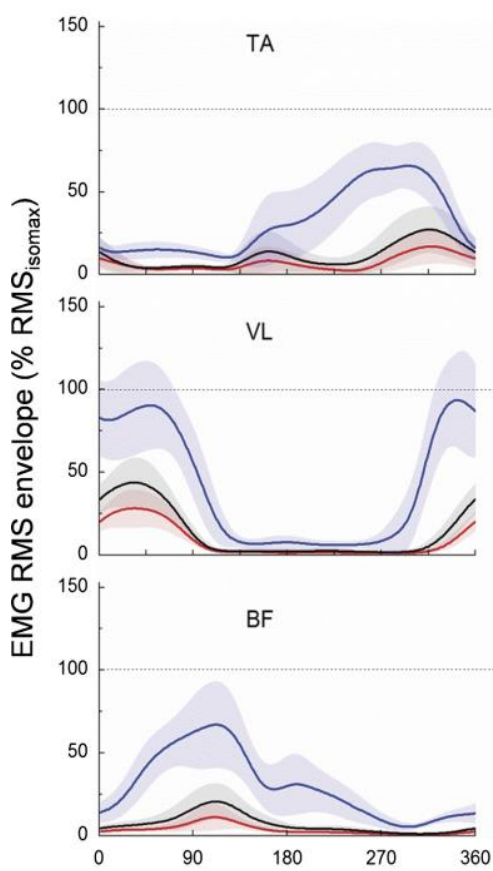

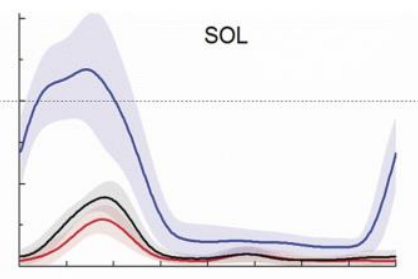

VM

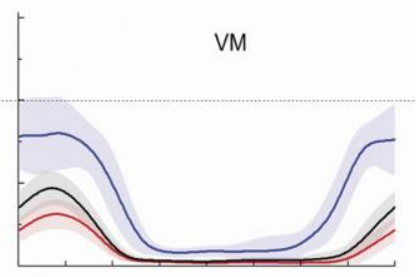

SM

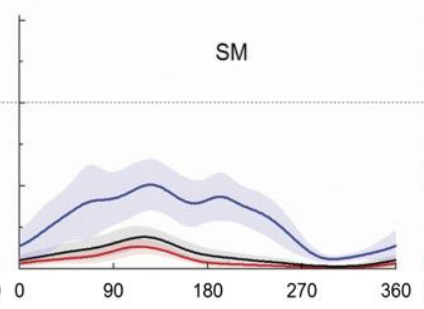

$\mathrm{GL}$

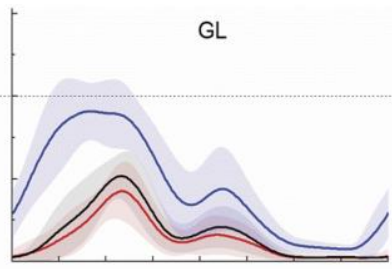

RF

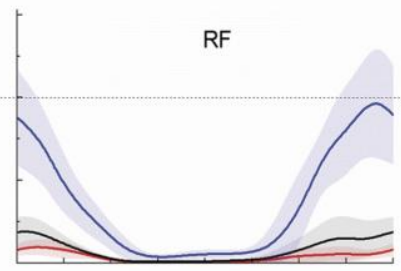

GMax

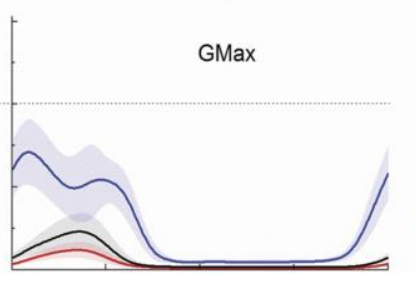

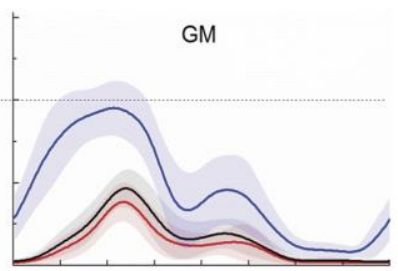

TF

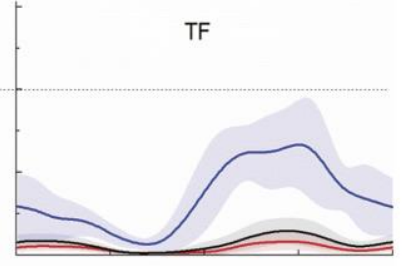

Crank torque

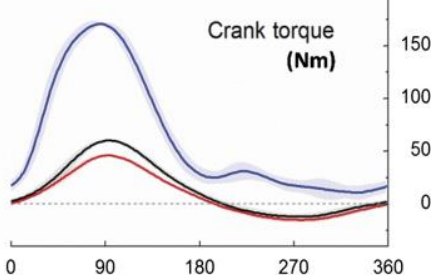

FIGURE 3-Ensemble-averaged EMG patterns of the 11 recorded muscles and crank torque profile (bottom right panel) for the two submaximal conditions (Sub150 W in red and SubVT2 in black) and the all-out sprint cycling (Sprint in blue) obtained on the 15 subjects (SD, intersubject variability). EMG and torque patterns were averaged across 30 (for Sub150 W and SubVT2) and 6-7 (for Sprint) consecutive pedaling cycles and expressed as a function of the crank position (highest pedal position: TDC $=0^{\circ}$ ). See Figure 2 for nomenclature of muscles. For color version, please refer to the online version at www.acsm-msse.com.

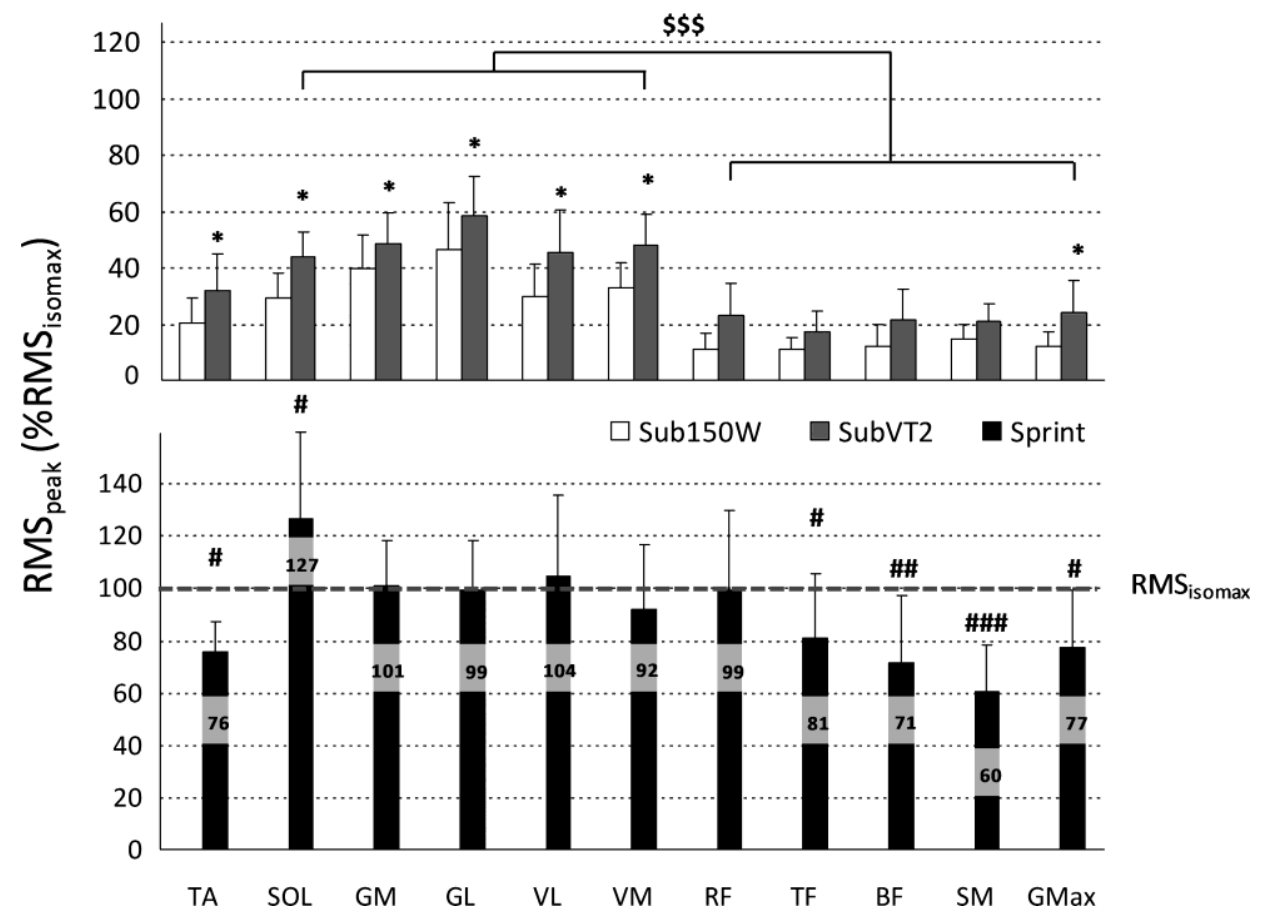

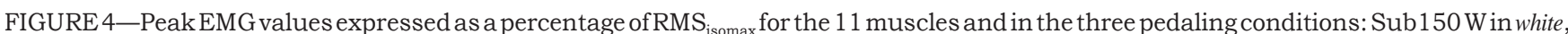
SubVT2 in gray (top panel), and Sprint in black (bottom panel). *Significant difference between Sub150 W and SubVT2 $(P<0.05)$. \$\$ Significant difference between the muscle groups $(P<0.001$, for both Sub150 W and SubVT2). Significant difference between Sprint and RMS isomax $(\# P<0.05$, $\# \# P<0.01$, \#\#\#P<0.001). See Figure 2 for nomenclature of muscles. 


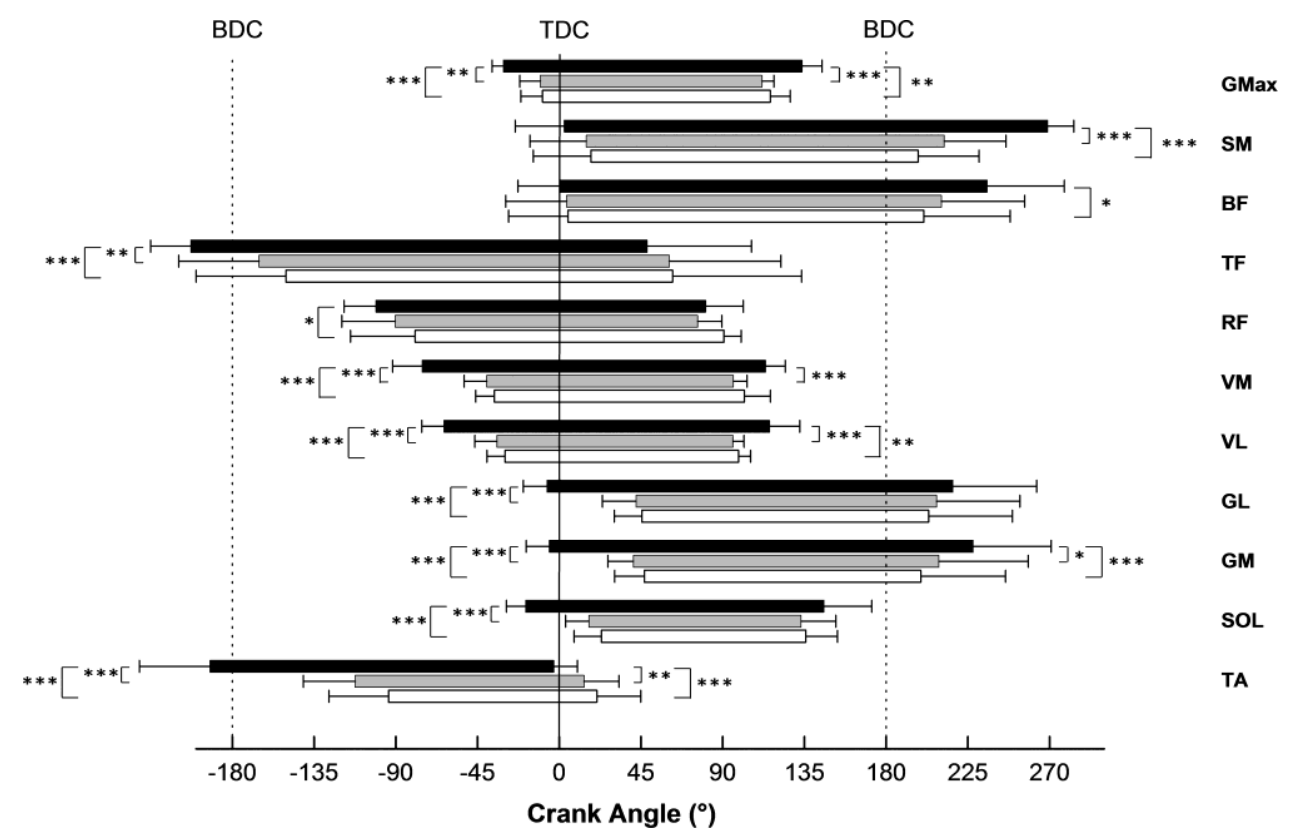

FIGURE 5-Mean onset, offset and duration of the burst of EMG activity indicated by bars for the 11 muscles, for the two submaximal conditions (Sub150 W in white and SubVT2 in gray) and the all-out sprint cycling (Sprint in black). Values are displayed as a function of crank position: $\operatorname{TDC}\left(0^{\circ}\right)$; $\mathrm{BDC}\left(180^{\circ}\right)$. Only the main burst is represented when two distinct bursts were observed for some muscles and some subjects. **** $P<0.001$, $* * P<0.01, * P<0.05$ significant differences between conditions.

during all-out sprint compared with submaximal conditions. More precisely, the onset of activation occurred earlier for all of the muscles (ranged from $P<0.01$ to $P<0.001$ ), except for BF and SM, and the offset occurred later (ranged from $P<0.05$ to $P<0.001$ ) for TA, GM, VL, VM, SM, GMax, and BF (Figs. 3 and 5). The burst duration expressed as the degree of crank rotation was significantly higher in sprint condition for TA, SOL, GM, GL, VL, VM, SM, and
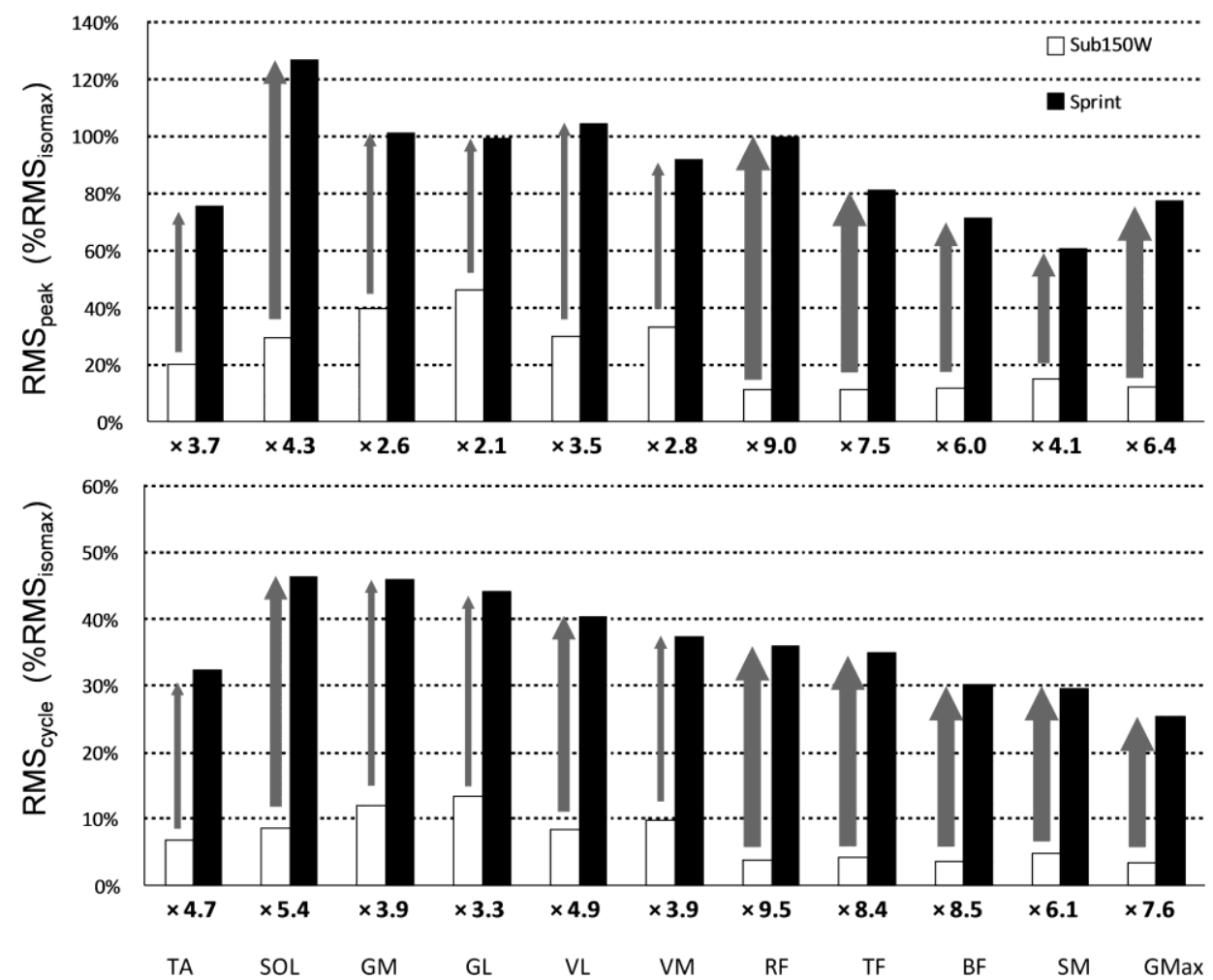

FIGURE 6-Rise of EMG activity between Sub150 W and Sprint condition (peak value: $\mathrm{RMS}_{\text {peak }}$ and mean value over the crank cycle: RMS $\mathrm{S}_{\text {cyle }}$ ). The thickness of the arrow is related to the magnitude of EMG increase in Sprint, referring to the Sub150 W condition ( $x n: n$-fold higher EMG activity in Sprint compared with Sub 150 W): small, from x1 to x4; medium, from $\times 4$ to $x 6$; large, higher than $x 6$. 
GMax $(P<0.01$ to $P<0.001)$ and was unchanged for RF, $\mathrm{TF}$, and $\mathrm{BF}$.

\section{DISCUSSION}

The purpose of this study was to investigate the alteration of muscle coordination during an all-out sprint cycling task compared with submaximal exercises. We aimed to compare changes in EMG activity between muscles and determine whether maximal muscle activation is reached during an allout sprint cycling task. Referring to the submaximal 150-W condition, the data showed that all-out sprint induced a higher increase of the mean $\left(\mathrm{RMS}_{\text {cycle }}\right)$ and peak $\left(\mathrm{RMS}_{\text {peak }}\right)$ level of activity for hip muscles (extensors and flexors) and knee flexors compared with knee and ankle extensors. In addition, the large increase of the period of activity for the majority of muscles during Sprint contributed to enlarge $\mathrm{RMS}_{\text {cycle. }} \mathrm{RMS}_{\text {peak }}$ values provide evidence that EMG activity level in Sprint, despite its large increase compared with the submaximal condition, does not grow to be systematically maximal for all muscles involved, especially for the thigh biarticular muscles (BF and SM) and the monoarticular hip extensor (GMax).

During submaximal exercises, monoarticular knee extensors (VL and VM) and plantar flexors (GM, GL, and SOL) were the most activated lower limb muscles and were those demonstrating a significant increase of their activity level from Sub150 W to the SubVT2 condition. In parallel, an absence of change in the activation timing for all of the muscles was observed between the two submaximal conditions (Figs. 2 and 4). Overall, the present data reinforced the statement $(9,18)$ that the control of power output during the submaximal cycling is clearly characterized by a major implication of knee extensors and ankle plantar flexors (and a control of their activity level), whereas the other muscles (especially the knee flexors and hip flexors) are weakly activated (almost $11 \%$ to $15 \%$ of $\mathrm{RMS}_{\text {isomax }}$ in the present study at $150 \mathrm{~W}$ ). The implication of hip extensor appears to be more debatable because the relatively low activity level of GMax seems to be not consistent with the results of Elmer et al. (8) who reported that hip extension was the dominant power-producing action during submaximal pedalling. Nevertheless, because EMG activity level is not directly related to force production, it remains difficult to make a precise association between the activity level (what we report herein) and the torque production at the level of the joints (results reported by Elmer and collaborators), without additional information about muscle architecture (e.g., PCSA) and the lever arms. In addition, the specificity of our population (highly trained cyclists with large PCSA) could partially be responsible to this relatively low level of activity of GMax despite a nonnegligible power-producing action of the hip extensors is expected. Furthermore, we did not record some other major hip extensors such as gluteus medius. Finally, the fact that GMax also demonstrated a significant increase of its activity from Sub $150 \mathrm{~W}$ to the SubVT2 condition supports its important implication in the control of power output during the submaximal cycling.

All-out pedaling sprint induced a power production almost nine times higher than that observed for Sub150 W $(1372$ vs. $150 \mathrm{~W})$. One of the main results was the discrepancy observed in the increase of the EMG peak value $\left(\mathrm{RMS}_{\text {peak }}\right)$ among muscles in this Sprint condition: seven to nine times higher activity for RF and TF; four to seven times higher activity for BF, SM, and GMax; and two to three times higher activity for VL, VM, GM, and GL (Fig. 6) than that in Sub150 W condition. This result highlights the larger involvement of the knee flexors and the hip extensors/ flexors during sprint compared with submaximal exercises. This result contradicts partly the suggestion made by some authors that the relative contribution of each lower limb muscles is conserved between maximal sprint and submaximal conditions $(10,24)$. Concerning the timing aspects of the coordination, individual EMG patterns demonstrated interesting specificities during all-out sprint condition: Adjustments in their shape over the crank cycle (Fig. 3) lead to an earlier onset and/or a later offset for the majority of the muscles and hence resulted in a longer period of activity compared with the submaximal conditions (Fig. 5). These results corroborate the proposition of Hug et al. (16) who reported alterations in synergy activation coefficients (which represent the relative contribution of muscle synergy to the overall muscle activity pattern throughout the crank cycle), suggesting some changes in timing of muscle activation between comparable submaximal and maximal pedaling conditions. However, they also disagree with the previous statement that muscles are activated at the same crank angular sector in submaximal and supramaximal cycling tasks $(10,24)$.

This important rise in the burst duration (in degrees of crank rotation) could serve as a means to maximize the action of the monoarticular extensor muscles (VM, VL, SOL, and GMax) throughout the crank angular sector corresponding to the extension phase of the joint (i.e., duty cycle as defined by Martin and Brown [21]). This adaptation is in good agreement with kinematic results obtained by Elmer et al. (8) who reported an earlier beginning but also a higher value of this duty cycle during sprint for the three joints (hip, knee, and ankle). For example, it is interesting to note in the all-out sprint condition, there is a match between the period of activity of $\mathrm{VM}$ and $\mathrm{VL}\left(189^{\circ}\right.$ and $179^{\circ}$, respectively) and the duty cycle for the knee reported by Elmer et al. $\left(185^{\circ}\right)$, whereas in submaximal condition, a real discrepancy was observed between these values (only $136^{\circ}$ and $130^{\circ}$ for VM and VL, respectively, with a duty cycle $=175^{\circ}$ ). For the biarticular muscles (SM, BF, GL, and GM), this interpretation is consistent as well, but the longer burst duration could also be understood as a way to improve the contribution of the second function attributed to these muscles (i.e., knee flexion in this case). For example, the latest offset and the alteration in the shape observed for SM, BF, and to a lesser 
extent, GL (i.e., a more consistent or little increase of activity specifically around and after BDC, Figs. 3 and 5) seem to indicate a higher implication altogether as knee flexor. Overall, these timing adaptations can be considered as an interesting instinctive coordination strategy (in addition to the increase of the peak activity level) to further enhance $\mathrm{RMS}_{\text {cycle }}$ (total quantity of EMG activity over the complete crank cycle) during supramaximal exercise for all of the muscles and particularly for those that are maximally or quasimaximally activated (i.e., $\mathrm{RMS}_{\text {peak }}$ value near 100\%). It is the case for GM, GL, and SOL increasing by about $47 \%$ their period of activity and for VM and VL increasing by about $38 \%$. Consequently, this adaptation certainly also has a critical role for increasing the mean muscle force produced over the complete cycle by the majority of muscles and thus the capacity to generate very higher mean crank torque in all-out sprint compared with the submaximal condition.

Even if it is difficult to directly infer the magnitude of the joint moment produced by a muscle basing only on its mean activity level, $\mathrm{RMS}_{\text {cycle }}$ data (Fig. 6) clearly support recent mechanical results about specific power of the joints $(6,8,21)$. More precisely, the larger increase in $\mathrm{RMS}_{\text {cycle }}$ observed in hamstrings (BF and SM) compared with vastii (VL and VM) is additional evidence of the relative higher knee flexion power and lower knee extension power (expressed as a percentage of total power over the complete cycle) measured in sprint cycling compared with submaximal exercise $(8,21)$. It is in accordance with the analysis of torque profile in the present study, showing a slight increase in the first part of the upstroke phase (between 190- and 240-) in the all-out sprint condition (Fig. 3). The very large increase of activity of the third knee extensor RF in all-out condition (i.e., 9-10 times higher than that in Sub150 W) could be explained by its biarticular function. We can reasonably assume that its action as hip flexor is certainly the primary factor responsible of such a very high increase in the activity of this muscle in sprint condition compared with VL and VM. It is also the case for BF and SM biarticular muscles also acting as hip extensor (in addition to their knee flexion action). Therefore, their $\mathrm{RMS}_{\text {cycle }}$ increase associated with the large increase of GMax activity is in a good agreement with the observation that hip extension is the most powerful action in the sprint condition (8,21). Finally, although Elmer et al. (8) reported no change in hip flexion power during sprint compared with a submaximal exercise, we found a large increase of $\mathrm{RMS}_{\text {peak }}$ in the hip flexors (TF and RF, Fig. 6). This discrepancy between EMG and mechanical data could be attributed to differences regarding the populations between both studies (trained endurance cyclists vs. top level sprint cyclists in the present study). However, it should be kept in mind that cycling posture (flexed forward truck) induced a necessary critically shortened configuration of the hip flexors with respect to the forcelength relation, preventing them from producing a high power (in contrast with other muscles such as VL [1]).

One of the original aims of this work was to use different MVC procedures to normalize muscle activity levels (i.e., isometric and isokinetic MVCs) and to provide information about the degree of muscle activation (in addition to the comparison with submaximal cycling conditions). Because of its lack of specificity in terms of posture, joint angle, muscle length, and/or type of contraction, the classical method based on isometric MVC had been sometimes criticized $(13,15,24)$. In the present study, we used a Con-Trex dynamometer allowing us to optimize the posture and angle in isometric mode $\left(\mathrm{MVC}_{\text {Isom }}\right)$ and to also apply the isokinetic mode $\left(\mathrm{MVC}_{\text {Isokin }}\right)$ to take into account the putative effect of the joint angle and muscle length on the maximal EMG response $(3,24)$. Moreover, the fact that our population was familiarized with both strength training and supramaximal exercises was a benefit to obtain maximal involvement and hence EMG activity level (13). Although this robust MVC procedure was carried out to estimate a maximal level of activity for each muscle group, it remains difficult to affirm that it completely avoids some underestimation of maximal EMG during isometric or isokinetic MVCs. It could, for instance, be an explanation of the typical high $\mathrm{RMS}_{\text {peak }}$ obtained for SOL in sprint condition (i.e., $126 \% \mathrm{RMS}_{\text {isomax }}$ ). In fact, using additional data obtained during the same session on 8 of the 15 subjects, we found an $\mathrm{RMS}_{\text {peak }}$ of $103 \%$ for SOL when referring to a maximal value obtained during a specific jumping squat with additional load (50\% of body mass). Note that this procedure did not modify maximal EMG activity of VL, VM, GL, and GM. Despite this limitation, it is noteworthy that the normalization procedure used in the present study has been recently reaffirmed as the most rigorous and reliable technique to provide information of how active a muscle in relation to its maximal activation capacity is $(3,17)$.

Normalized EMG activity levels (in percentage of $\mathrm{RMS}_{\text {isomax }}$ ) only partially support our initial hypothesis that all-out sprint cycling would allow all muscles to be maximally recruited. In fact, although the muscles from quadriceps and triceps surae demonstrated maximal $\mathrm{RMS}_{\text {peak }}$ value, hip extensors and knee flexors (GMax, BF, SM) and hip (TF) and dorsi (TA) flexors showed values substantially lower $\left(60 \%-80 \%\right.$ of $\left.\mathrm{RMS}_{\text {isomax }}\right)$. Therefore, the assumption that such brief all-out cycling exercise requires maximal or nearmaximal muscle recruitment $(4,28)$ is clearly confirmed for some of the main power producers (i.e., quadriceps muscles) and muscles responsible of the transfer to the crank of the limb segment energy generated by the hip and knee extensors (i.e., triceps surae $[23,33])$. However, it is not confirmed for the other main power producers (hip extensors), the muscles in charge of power transfer and/or optimization of the pedal force orientation (hamstrings), and the lower power producers and/or transmitters (hip and dorsi flexors, $\mathrm{TF}$, and TA).

Although some studies suggested to use an all-out sprint pedaling exercise for EMG normalization $(10,24)$, the pertinence of such a procedure has been questioned $(13,15)$. Data reported in the current study provide experimental evidence that this procedure can lead to some misinterpretations. In fact, 
muscle activity level achieved during an all-out pedaling sprint fails to systematically reflect the maximal neural drive of the different lower limb muscles. Furthermore, one would expect that untrained subjects would have demonstrated an even lower activity level and a higher intersubject variability. Finally, these authors $(10,24)$ justified the pertinence of using sprint test procedure arguing similarities in the contribution of the different muscles, their timing of activation and the joint angles between submaximal and all-out sprint pedaling, without providing any experimental data to support that. The results of the present study as discussed in the previous part allow us to demonstrate that all the statements are unfortunately not verified. Thus, in agreement with the recent review of Burden (3), we believe that the normalization methods based on sprint cycling should not be systematically recommended (instead of MVC procedure) when the aim is i) to provide information on how active a muscle is in relation to its maximal activation capacity or ii) to facilitate comparison between EMGs for different muscles and submaximal pedaling conditions (especially for some biarticular muscles, BF and SM, and also monoarticular GMax).

Overall, the present results give interesting information to researchers and clinicians regarding the recruitment of the different muscle groups. It is particularly appropriate not only for studies using the supramaximal pedaling task as a useful paradigm to induce fatigue and investigate associated metabolic, neurophysiological, and/or biomechanical adaptations on a specific muscle group (the vastii most of the time), but also for clinicians and coaches wishing to train definite muscle groups. From a sport performance point of view, these results provide perspectives to optimize the maximal power production in sprint cyclists (track and road) by training interventions. The very high level of activity of quadriceps and even more of triceps surae seems to indicate that, independently of the neuromuscular aspects, the intrinsic properties associated with maximal strength ability of these two muscle groups (in reference to their physiological cross-sectional area) remain of primary importance to expect an improvement of the force exerted on the pedal. As mentioned above, triceps surae being responsible of transferring force produced by proximal muscles groups (hip extensors and knee extensors) to the pedal, one would expect that strength ability of these muscles groups could be considered as a limitation factor that prevents a higher recruitment of more powerful muscles such as glutei. The no-maximal recruitment of biarticular hamstring muscles and the important variability observed in the duration of their activity between the subjects (even in this expert population, Fig. 5) question the pertinence of specific training to improve the ability to activate these muscles as high as possible during a crank

\section{REFERENCES}

1. Austin N, Nilwik R, Herzog W. In vivo operational fascicle lengths of vastus lateralis during sub-maximal and maximal cycling. J Biomech. 2010;43(12):2394-9. cycle period as long as possible during the upstroke phase (i.e., to pull up the pedal more effectively). On the whole, all of these qualitative and quantitative alterations of coordination are certainly a main explanation of the great mechanical effectiveness reported during the sprint task (i.e., ability to efficiently orientate the total force on the pedal; ratio between effective force and total force ranged from $75 \%$ to $80 \%$ reported by Dorel at al. [6]). Finally, these results clearly substantiate recent hypotheses concerning the general understanding of the role of muscle coordination in the external power production during these kinds of multijoint tasks $(19,31)$, especially those stating that, during this type of all-out sprint cycling tasks, intermuscular coordination is a key factor contributing to limiting global power output and explaining differences that could be related to age (19), to maximum power from any one muscle itself (31), and hence to the level of expertise of the subject.

In conclusion, the present study is the first to report changes in EMG activity level associated to an all-out sprint pedaling task in comparison with submaximal exercises. We showed that an all-out sprint is characterized by a very large increase in EMG activity level for the hip flexors (multiplied by 7 to 9 from $150 \mathrm{~W}$ to sprint condition) and, to a lesser extent, for the knee flexors and hip extensors (multiplied by 5 to 7), whereas plantar flexors and knee extensors demonstrate a lower increase (multiplied by 2 to 3 ). This result clearly suggests a change in the relative contribution of the different muscles to the power production between submaximal and maximal cycling tasks. During an all-out sprint, EMG activity level failed to reach a maximal value for some muscles, especially for hamstrings and GMax (i.e., < $70 \%$ to $80 \%$ of peak EMG activity during MVC), putting forward precautions in the use of this supramaximal pedaling task as a reference normalization condition. Finally, an important increase in the duration of activity during all-out sprint is likely to represent an interesting coordination strategy to enhance the work generated by all of the muscle groups. Further studies are needed to investigate whether this coordination could be optimized by training procedures, to increase the pedaling effectiveness and hence the power output with the hope to induce significant gains in cycling sprint performance.

This study was funded in part by French Ministry of Sport: contract 10-R-019.

The authors are grateful to the subjects for having agreed to participate in this study and to the national head coaches in track cycling and the French Federation of Cycling. The authors report no conflict of interest.

The results of the present study do not constitute endorsement by the American College of Sports Medicine.

2. Baum BS, Li L. Lower extremity muscle activities during cycling are influenced by load and frequency. J Electromyogr Kinesiol. 2003;13(2):181-90. 
3. Burden A. How should we normalize electromyograms obtained from healthy participants? What we have learned from over 25 years of research. J Electromyogr Kinesiol. 2010;20(6):1023-35.

4. Dorel S, Bourdin M, Van Praagh E, Lacour JR, Hautier CA. Influence of two pedalling rate conditions on mechanical output and physiological responses during all-out intermittent exercise. Eur J Appl Physiol. 2003;89(2):157-65.

5. Dorel S, Couturier A, Hug F. Influence of different racing positions on mechanical and electromyographic patterns during pedalling. Scand J Med Sci Sports. 2009;19(1):44-54.

6. Dorel S, Couturier A, Lacour JR, Vandewalle H, Hautier C, Hug F. Force-velocity relationship in cycling revisited: benefit of twodimensional pedal forces analysis. Med Sci Sports Exerc. 2010; 42(6):1174-83.

7. Dorel S, Drouet JM, Couturier A, Champoux Y, Hug F. Changes of pedaling technique and muscle coordination during an exhaustive exercise. Med Sci Sports Exerc. 2009;41(6):1277-86.

8. Elmer SJ, Barratt PR, Korff T, Martin JC. Joint-specific power production during submaximal and maximal cycling. Med Sci Sports Exerc. 2011;43(10):1940-7.

9. Ericson M. On the biomechanics of cycling. A study of joint and muscle load during exercise on the bicycle ergometer. Scand $J$ Rehabil Med Suppl. 1986;16:1-43.

10. Fernandez-Pena E, Lucertini F, Ditroilo M. A maximal isokinetic pedalling exercise for EMG normalization in cycling. $J$ Electromyogr Kinesiol. 2009;19(3):e162-70.

11. Hautier CA, Arsac LM, Deghdegh K, Souquet J, Belli A, Lacour JR. Influence of fatigue on EMG/force ratio and cocontraction in cycling. Med Sci Sports Exerc. 2000;32(4):839-43.

12. Hermens HJ, Freriks B, Disselhorst-Klug C, Rau G. Development of recommendations for SEMG sensors and sensor placement procedures. J Electromyogr Kinesiol. 2000;10(5):361-74.

13. Hug F. Can muscle coordination be precisely studied by surface electromyography? J Electromyogr Kinesiol. 2011;21(1):1-12.

14. Hug F, Bendahan D, Le Fur Y, Cozzone PJ, Grelot L. Heterogeneity of muscle recruitment pattern during pedaling in professional road cyclists: a magnetic resonance imaging and electromyography study. Eur J Appl Physiol. 2004;92(3):334-42.

15. Hug F, Dorel S. Electromyographic analysis of pedaling: a review. J Electromyogr Kinesiol. 2009;19(2):182-98.

16. Hug F, Turpin NA, Couturier A, Dorel S. Consistency of muscle synergies during pedaling across different mechanical constraints. J Neurophysiol. 2011;106(1):91-103.

17. Hunter AM, St Clair Gibson A, Lambert M, Noakes TD. Electromyographic (EMG) normalization method for cycle fatigue protocols. Med Sci Sports Exerc. 2002;34(5):857-61.
18. Jorge M, Hull ML. Analysis of EMG measurements during bicycle pedalling. J Biomech. 1986;19(9):683-94.

19. Korff T, Hunter EL, Martin JC. Muscular and non-muscular contributions to maximum power cycling in children and adults: implications for developmental motor control. J Exp Biol. 2009; 212(Pt 5):599-603.

20. Laplaud D, Hug F, Grelot L. Reproducibility of eight lower limb muscles activity level in the course of an incremental pedaling exercise. J Electromyogr Kinesiol. 2006;16(2):158-66.

21. Martin JC, Brown NA. Joint-specific power production and fatigue during maximal cycling. J Biomech. 2009;42(4):474-9.

22. Prilutsky BI. Coordination of two- and one-joint muscles: functional consequences and implications for motor control. Motor Control. 2000;4(1):1-44.

23. Raasch CC, Zajac FE, Ma B, Levine WS. Muscle coordination of maximum-speed pedaling. J Biomech. 1997;30(6):595-602.

24. Rouffet DM, Hautier CA. EMG normalization to study muscle activation in cycling. J Electromyogr Kinesiol. 2008;18(5):866-78.

25. Rouffet DM, Mornieux G, Zameziati K, Belli A, Hautier CA. Timing of muscle activation of the lower limbs can be modulated to maintain a constant pedaling cadence. J Electromyogr Kinesiol. 2009;19(6):1100-7.

26. Samozino P, Horvais N, Hintzy F. Why does power output decrease at high pedaling rates during sprint cycling? Med Sci Sports Exerc. 2007;39(4):680-7.

27. Sanderson DJ, Black A. The effect of prolonged cycling on pedal forces. J Sports Sci. 2003;21(3):191-9.

28. Sargeant AJ. Human power output-determinants of maximum performance. In: Human Muscular Function during Dynamic Exercise; Marconnet P, Saltin B, Komi P, Poortmans J, editors. Medicine Sports Science, Basel: Karger; 1996. p. 10-20.

29. Sarre G, Lepers R, Maffiuletti N, Millet G, Martin A. Influence of cycling cadence on neuromuscular activity of the knee extensors in humans. Eur J Appl Physiol. 2003;88(4-5):476-9.

30. Ting L, Chvatal S. Decomposing muscle activity in motor tasks: methods and interpretation. In: Danion F, Latash M, editors. Motor Control. Oxford: Oxford Univ. Press; 2010. p. 103-38.

31. Wakeling JM, Blake OM, Chan HK. Muscle coordination is key to the power output and mechanical efficiency of limb movements. J Exp Biol. 2010;213(3):487-92.

32. Weijs WA, Sugimura T, van Ruijven LJ. Motor coordination in a multi-muscle system as revealed by principal components analysis of electromyographic variation. Exp Brain Res. 1999;127(3):233-43.

33. Zajac FE. Understanding muscle coordination of the human leg with dynamical simulations. J Biomech. 2002;35(8):1011-8. 